\title{
Effects of doxorubicin and mitoxantrone in the brain of differently aged mice: an in vivo chemobrain study
}

$\underline{\text { Ana Dias-Carvalho }}^{1}$, Ana Reis-Mendes ${ }^{1}$, Margarida Duarte-Araújo ${ }^{2,3}$, Félix Carvalho ${ }^{1}$, Maria Lourdes Bastos ${ }^{1}$, Susana I. Sá ${ }^{4,5}$, João Paulo Capela ${ }^{1,6}$ and Vera Marisa Costa ${ }^{1}$

${ }^{1}$ UCIBIO, REQUIMTE, Laboratory of Toxicology, Department of Biological Sciences, Faculty of Pharmacy, University of Porto, Porto, Portugal; ${ }^{2}$ LAQV/REQUIMTE, University of Porto, Porto, Portugal; ${ }^{3}$ Department of Imuno-Physiology and Pharmacology, Institute of Biomedical Sciences Abel Salazar, University of Porto, Portugal; ${ }^{4}$ Faculty of Medicine, Department of Anatomy University of Porto, Porto, Portugal. ${ }^{5}$ Faculty of Medicine, Center for Health Technology and Services Research (CINTESIS), University of Porto, Porto, Portugal; ${ }^{6}$ FP-ENAS (Unidade de

Investigação UFP em Energia, Ambiente e Saúde), CEBIMED (Centro de Estudos em Biomedicina), Faculdade de Ciências da Saúde, Universidade Fernando Pessoa, Porto, Portugal

Introduction

Despite its success in cancer-treatment, chemotherapy targets healthy tissues, which leads to toxicity and long-term health problems (1). The term "chemobrain" is used to summon the cognitive deficit effects of chemotherapy in the long term (2). Chemobrain affects $\mathbf{1 7 \%}$ to $\mathbf{3 4 \%}$ of chemotherapy-treated patients (2). Doxorubicin (DOX) and mitoxantrone (MTX) are two widely used chemotherapeutic agents with a broad spectrum of activity against neoplastic cells (3). Therefore, this work aimed to evaluate the effects towards the brain of clinically relevant doses of DOX and MTX in male CD-1 mice of different ages (infant, adult, old), and to understand if these pathways are involved in DOX and MTX-induced chemobrain.

Experimental Protocol

\begin{tabular}{|c|c|c|c|c|}
\hline & Age & Average weight (g) & $\begin{array}{c}\text { Total cumulative } \\
\text { dose of DOX }\end{array}$ & $\begin{array}{c}\text { Total cumulative } \\
\text { dose of } \text { MTX }\end{array}$ \\
\hline Infant & 4 weeks & 23 & \multirow{2}{*}{$18 \mathrm{mg} / \mathrm{kg}$} & \multirow{2}{*}{$6 \mathrm{mg} / \mathrm{kg}$} \\
\cline { 1 - 3 } Adult & 3 months & 47 & & \\
\hline Old & $18-20$ months & 63 & $9 \mathrm{mg} / \mathrm{kg}$ & \\
\hline
\end{tabular}

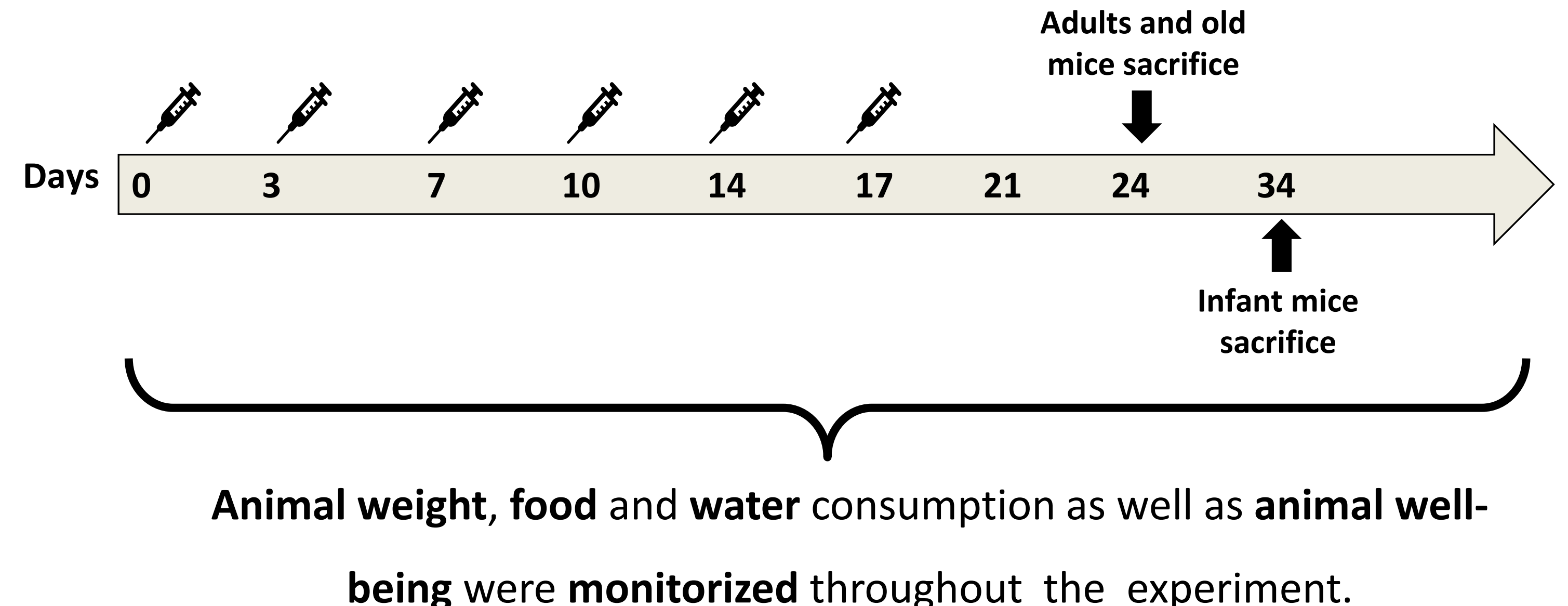

Sections are being used for immunofluorescent detection of phosphorylated Tau, glial fibrillary acidic protein, BAX and p53 proteins in the hippocampal formation.

Adult left-brain hemisphere was fixated in $4 \%$ paraformaldehyde.

\section{Results}

\begin{tabular}{|c|c|c|c|c|c|c|}
\hline \multirow[b]{2}{*}{ Treatment } & \multicolumn{2}{|c|}{ Juvenile } & \multicolumn{2}{|c|}{ Adult } & \multicolumn{2}{|c|}{ Old } \\
\hline & DOX & MTX & DOX & MTX & DOX & MTX \\
\hline Average body weight & *Decreased & No changes & ${ }^{*}$ Decreased & No changes & No changes & No changes \\
\hline GSH total & \multirow{4}{*}{ No changes } & \multirow{4}{*}{ No changes } & ${ }^{*}$ Decreased & \multirow[t]{4}{*}{ No changes } & \multirow[t]{4}{*}{ No changes } & \multirow[t]{4}{*}{ No changes } \\
\hline GSH & & & No changes & & & \\
\hline GSSG & & & *Decreased & & & \\
\hline GSH/GSSG & & & *Decreased & & & \\
\hline ATP & *Increased & No changes & No changes & No changes & \multicolumn{2}{|c|}{ Not quantified } \\
\hline
\end{tabular}
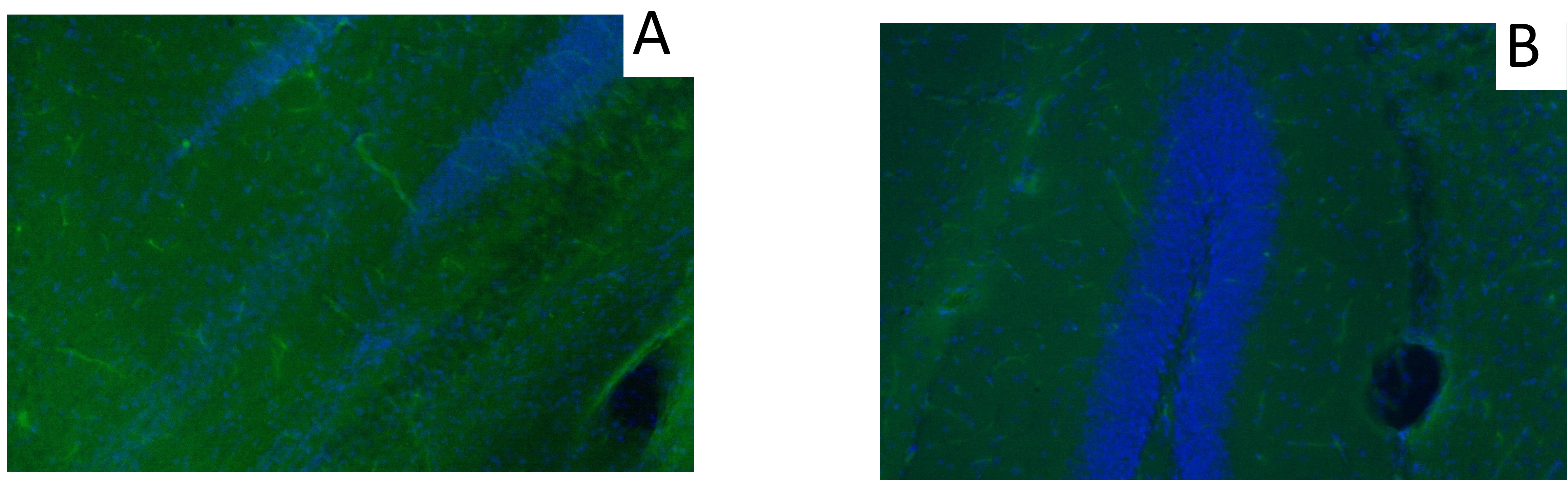

*Comparing to the respective control group.

B

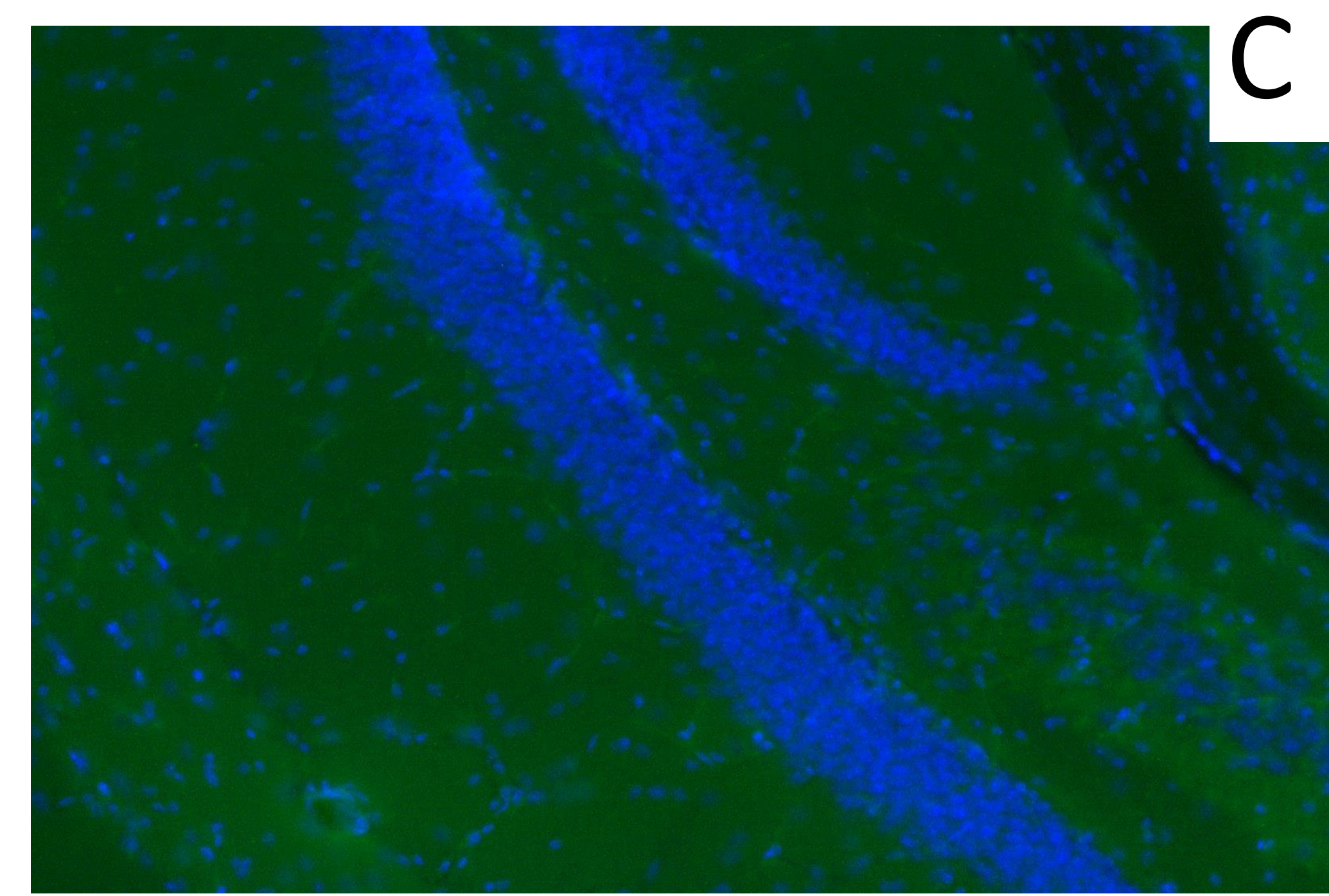

Figure 1: Fluorescence microscopy images of the dentate gyrus stained with Alexa Fluor 488 (green, phosphorylated tau) and the cell nuclei stained with DAPI (blue) in DOX $18 \mathrm{mg} / \mathrm{kg}$ treated mice ( $\mathrm{A}$ to C).

\section{Discussion and conclusions}

Considering the measurements of the glutathione levels as a marker of oxidative stress, only the adult mice treated with DOX had significant alterations namely lower levels of GSHt and GSH and a decrease in the GSH/GSSG ratio. The administration of DOX also increased the ATP levels in the infant mice. The MTX-treatment did not affect the measured parameters in any groups tested. The presented data suggests that DOX causes redox impairment and could be a possible cause of chemobrain in the adult brain. On the other hand, MTX was shown not to influence the GSH levels and cause less distress in this dose to the animals.

\section{References:}

(1) Simó M, Rifà-Ros X, Rodriguez-Fornells A, Bruna J. Chemobrain: a systematic review of structural and fun

(2) Seiter K. Toxicity of the topoisomerase II inhibitors. Expert opinion on drug safety. 2005;4(2):219-34. 